\title{
Palladium- and Copper-Catalyzed Amination of Halogenophenyl Substituted Porphyrins for the Synthesis of Porphyrin-Azacrown Ethers Conjugates and Evaluation of Their Sensing Properties
}

\author{
Alexei A. Yakushev, ${ }^{a}$ Alexei D. Averin, ${ }^{\mathrm{a}, \mathrm{b} @}$ Olga A. Maloshitskaya, ${ }^{\mathrm{b}}{ }$ Sergei A. Syrbu, ${ }^{\mathrm{c}}$ \\ Oskar I. Koifman, ${ }^{\mathrm{c}}$ and Irina P. Beletskaya ${ }^{\mathrm{a}, \mathrm{c}}$
}

\section{Dedicated to Academician of Russian Academy of Sciences Oleg. G. Sinyashin} on the occasion of his $60^{\text {th }}$ Birthday

\begin{abstract}
${ }^{a}$ A.N. Frumkin Institute of Physical Chemistry and Electrochemistry, 119991 Moscow, Russian Federation
${ }^{\mathrm{b}}$ Lomonosov Moscow State University, Department of Chemistry, 119991 Moscow, Russian Federation

'Ivanovo State University of Chemistry and Technology, 153000 Ivanovo, Russian Federation

${ }^{\circledR}$ Corresponding author E-mail: alexaveron@yandex.ru
\end{abstract}

\begin{abstract}
The $P d^{0}$ - and $C u^{I}$-catalyzed amination of meso-(halogenophenyl)substituted porphyrins and their Zn complexes with propane-1,3-diamine and trioxadiamine was carried out to produce mono- and diaminated derivatives. Conditions for the synthesis of bis-and trismacrocyclic porphyrin-azacrown conjugates with trimethylenediamine linkers via $P d^{0}$-catalyzed amination reactions were established. The properties of two polymacrocyclic compounds to serve as colorimetric and fluorescent sensors for metal cations were investigated, and trismacrocyclic ligand possessing two 1-aza-18-crown-6 moieties arranged symmetrically around the porphyrin core was found to be selective for Cu in the presence of other metals by strong emission quenching caused by copper ions.
\end{abstract}

Keywords: Macrocycles, amination, Pd catalysis, $\mathrm{Cu}$ catalysis, porphyrins, azacrown ethers, conjugates, chemosensor.

\section{ПаллаАий- и меАь-катализируемое аминирование галогенфенил замещенных порфиринов в синтезе конъюгатов порфиринов с азакраун-эфирами и оценка их свойств как Аетекторов}

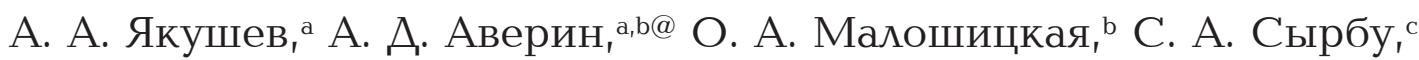

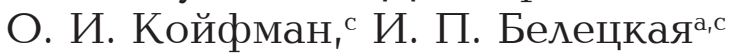
Посвящается Акаgемику РАН Олегу ГерольgовичуСиняшину по случаю его 60-летнего юбилея

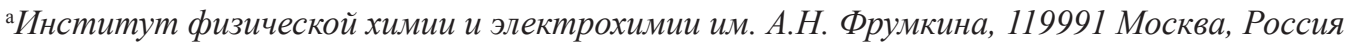

${ }^{\mathrm{b}}$ Московский государственный университет им. М.В. Ломоносова, Химический факультет, 119991 Москва, Россия

${ }^{\mathrm{c}}$ Ивановский государственный химико-технологический университет, 153000 Иваново, Россия

@E-mail: alexaveron@yandex.ru
}

$P d^{0}$ - и Сй-катализируемое аминирование мезо-(галогенфенил) замещченных порфиринов и их ичинковых комплексов с пропан-1,3-диамином и триоксадиамином привело кполучению моно- и диаминопроизводных. Найдены условия синтеза бис-и трисмакрочиклических конъюгатов с триметилендиаминовыми линкерами с использованием реакиий $P d^{0}$-катализируемого аминирования. Исследованы свойства двух полимакрочиклических производньх 
для колориметрического и флуориметрического определения катионов металлов, обнаружено, что трисмакроииклический лиганд с порфириновым двумя фрагментами 1-аза-18-краун-6 эфира, симметрично расположенными вокруг порфиринового ядра, селективен в отнотении катионов Си в присутствии остальньх металлов, и указанные ионь избирательно и полностью гасят флуоресценцию данного лиганда.

Ключевые слова: Макроциклы, аминирование, $\mathrm{Pd}$ катализ, $\mathrm{Cu}$ катализ, порфирины, азакраун-эфиры, конъюгаты, хемосенсоры на ионы металлов.

\section{Introduction}

Modification of porphyrins using catalytic approaches is well documented in literature. ${ }^{[1]}$ For example, mesohalogenophenyl substituted porphyrins are known to participate in the $\mathrm{Pd}^{0}$-mediated amination reactions. ${ }^{[2]}$ Much more extensive catalytic transformations have been described for meso-halogenosubstituted porphyrins: amination, ${ }^{[3,4]}$ alkoxylation and aryloxylation, ${ }^{[5,6]}$ sulfonylation, ${ }^{[7]}$ and phosphorylation. ${ }^{[8]}$ The formation of carbon-carbon bonds via Suzuki, Sonogashira and Heck reactions are the powerful tools for various modifications of porphyrins ${ }^{[9-11]}$ and construction of di- and triporphyrin systems of various architectures. ${ }^{[12-20]}$ All these reactions were accomplished using Pd catalysis. As for copper-mediated reactions in porphyrins transformation, the majority of literature data deals with "click" reactions which give a lot of possibilities of porphyrins modifications. [21-24] However, in other transformations copper plays a humble role; for example its application was described for the catalytic tetraarylation of meso-tetrakis(4-aminophenyl) porphyrin with 4-iodoanisole, ${ }^{[25]} \mathrm{CuCl}$ was employed for the synthesis of an awesome square-shaped $\pi$-conjugated porphyrin tetramer with diacetylene linkers, ${ }^{[26]} \mathrm{Cu}(\mathrm{OAc})_{2}$ was used in the synthesis of dibutadiyne-bridged porphyrin macrotricyclic dimer. ${ }^{[27]}$

Recently some of us have studied the amination of mono- and bis(bromophenyl)porphyrins using diamines with secondary amino groups like 1,2-dimethylethane-1,2diamine (DMEDA) and piperazine, ${ }^{[28]}$ these compounds have been used for the synthesis of porphyrin dyads and triads of various architecture. ${ }^{[29]}$ Copper-mediated famous "click" reactions were also used in the synthesis of porphyrin trimers. ${ }^{[30]}$ In the present work we studied various approaches using $\mathrm{Pd}$ - and $\mathrm{Cu}$-catalyzed amination reactions for conjugating porphyrin moiety with azacrown ethers, macrocyclic and macrobicyclic compounds in view of the synthesis of hybrid polymacrocyclic systems - novel polytopic ligands able to bind different metal cations.

\section{Experimental}

NMR spectra were registered using Bruker Avance 400 spectrometer, MALDI-TOF spectra were obtained with Bruker Autoflex II spectrometer using 1,8,9-trihydroxyanthracene as matrix and PEGs as internal standards. Propane-1,3-diamine, dioxa- and trioxadiamines, azacrown ethers, 2-(isobutyryl) cyclohexanone, BINAP, DavePhos, cesium carbonate, sodium tert-butoxide, $\mathrm{CuI}$ were purchased from Sigma-Aldrich and used without further purification, $\mathrm{Pd}(\mathrm{dba})_{2}$ was synthesized according to the method described, ${ }^{[31]}$ halogenophenylsubstituted porphyrines and their Zn complexes 1, 2, 9-12 were synthesized according to a described procedure, ${ }^{[32]}$ synthesis of 3-bromobenzyl derivatives of azacrown ethers $\mathbf{2 3}$ and $\mathbf{2 4}$ and preparation of diamino derivatives of benzylazacrown ethers $\mathbf{2 5}$ and $\mathbf{2 6}$ were carried out according to a known method. ${ }^{[33]}$ Dioxane was distilled over $\mathrm{NaOH}$ followed by the distillation over sodium under argon, acetonitrile was distilled over $\mathrm{CaH}_{2}, \mathrm{DMF}$, dichloromethane and methanol were used freshly distilled.

Porphyrin 5. A two-necked flask flushed with dry argon, equipped with a magnetic stirrer and reflux condenser was charged with porphyrin $1(0.11 \mathrm{mmol}, 83 \mathrm{mg})$, trioxadiamine $4(0.33 \mathrm{mmol}$, $73 \mathrm{mg}), \mathrm{Pd}(\mathrm{dba})_{2}(5 \mathrm{mg}, 8 \mathrm{~mol} \%)$, BINAP (6 mg, $9 \mathrm{~mol} \%$ ), absolute dioxane $(2 \mathrm{ml})$ and ${ }^{t} \mathrm{BuONa}(0.2 \mathrm{mmol}, 20 \mathrm{mg})$. The reaction mixture was refluxed for $24 \mathrm{hrs}$, cooled to ambient temperature, diluted with $\mathrm{CH}_{2} \mathrm{Cl}_{2}(5 \mathrm{ml})$, the residue was filtered off, washed with $\mathrm{CH}_{2} \mathrm{Cl}_{2}(5 \mathrm{ml})$, combined organic fractions were evaporated in vacuo, and chromatographed on silica gel using a gradient of eluents: $\mathrm{CH}_{2} \mathrm{Cl}_{2}-\mathrm{CH}_{2} \mathrm{Cl}_{2} / \mathrm{MeOH}$ 3:1 $-\mathrm{CH}_{2} \mathrm{Cl}_{2} / \mathrm{MeOH} / \mathrm{NH}_{3}$ aq 100:20:1-10:14:1. The target compound was eluted with $\mathrm{CH}_{2} \mathrm{Cl}_{2} /$ $\mathrm{MeOH} / \mathrm{NH}_{3}$ aq 100:20:3. Yield $16 \mathrm{mg}$ (16\%), deep-red solid. Mass spectrum (MALDI TOF) $m / z: 890.4753[\mathrm{M}]^{+} . \mathrm{C}_{52} \mathrm{H}_{70} \mathrm{~N}_{6} \mathrm{O}_{3} \mathrm{Zn} .{ }^{1} \mathrm{H}$ NMR $\left(\mathrm{CDCl}_{3}, 298 \mathrm{~K}\right) \delta_{\mathrm{H}} \mathrm{ppm}: 0.98\left(6 \mathrm{H}, \mathrm{t},{ }^{3} \mathrm{~J}=7.3 \mathrm{~Hz}\right), 1.55(4 \mathrm{H}$, sextet, ${ }^{3} J=7.4 \mathrm{~Hz}$ ), 1.75 (4H, quintet, $\left.{ }^{3} J=7.3 \mathrm{~Hz}\right), 1.90$ ( $2 \mathrm{H}$, br.s), $1.99\left(2 \mathrm{H}\right.$, quintet, $\left.{ }^{3} J=5.6 \mathrm{~Hz}\right), 2.27\left(4 \mathrm{H}\right.$, quintet, $\left.{ }^{3} J=7.5 \mathrm{~Hz}\right), 2.52$ (6H, s), 3.20 (2H, br.s), 3.39 (2H, br.s), 3.47 (6H, s), 3.49-3.60 (8H, $\mathrm{m}), 3.56(6 \mathrm{H}, \mathrm{s}), 3.62(2 \mathrm{H}$, br.s $), 3.68\left(2 \mathrm{H}, \mathrm{t},{ }^{3} \mathrm{~J}=5.4 \mathrm{~Hz}\right), 3.96(4 \mathrm{H}$, $\left.\mathrm{t},{ }^{3} \mathrm{~J}=7.3 \mathrm{~Hz}\right), 7.04\left(2 \mathrm{H}, \mathrm{d},{ }^{3} J_{o b s}=8.2 \mathrm{~Hz}\right), 7.72\left(2 \mathrm{H}, \mathrm{d},{ }^{3} J_{o b s}=8.2 \mathrm{~Hz}\right)$, $9.81(1 \mathrm{H}, \mathrm{s}), 9.96(2 \mathrm{H}, \mathrm{s}), \mathrm{NH}$ protons were not assigned.

Porphyrin 6. A two-necked flask flushed with dry argon, equipped with a magnetic stirrer and reflux condenser was charged with porphyrin $2(0.15 \mathrm{mmol}, 120 \mathrm{mg})$, propane-1,3-diamine (3) $(0.45 \mathrm{mmol}, 33 \mathrm{mg}), \mathrm{CuI}(3 \mathrm{mg}, 10 \mathrm{~mol} \%)$, 2-(isobutyryl) cyclohexanone $(6 \mathrm{mg}, 20 \mathrm{~mol} \%)$, DMF $(1 \mathrm{ml})$ and $\mathrm{Cs}_{2} \mathrm{CO}_{3}$ $(0.225 \mathrm{mmol}, 75 \mathrm{mg})$. The reaction mixture was stirred at $110^{\circ} \mathrm{C}$ for $24 \mathrm{hrs}$, cooled to ambient temperature, diluted with $\mathrm{CH}_{2} \mathrm{Cl}_{2}(5 \mathrm{ml})$, the residue was filtered off, washed with $\mathrm{CH}_{2} \mathrm{Cl}_{2}(5 \mathrm{ml})$, combined organic fractions were evaporated in vacuo to produce compound $\mathbf{6}$. Yield: $55 \mathrm{mg}$ (50\%), deep-red solid. Mass spectrum (MALDI TOF) $m / z: 744.3803[\mathrm{M}]^{+} .{ }^{1} \mathrm{H}$ NMR $\left(\mathrm{CDCl}_{3}, 298 \mathrm{~K}\right) \delta_{\mathrm{H}}$ ppm: $0.98(6 \mathrm{H}$, br.s), 1.55 (4H, br.s), 1.71 (4H, br.s), 2.03 (2H, br.s), 2.30 (4H, br.s), 2.45 (6H, br.s), 2.57 (2H, br.s), 3.48-3.58 (14H, br.m), $6.34(1 \mathrm{H}$, br.s), $6.70(1 \mathrm{H}$, br.s), 7.20 (1H, br.s), 7.35 (H, br.s), NH protons were not assigned.

Porphyrin 7. A two-necked flask flushed with dry argon, equipped with a magnetic stirrer and reflux condenser was charged with porphyrin $2(0.15 \mathrm{mmol}, 120 \mathrm{mg})$, trioxadiamine $4(0.3 \mathrm{mmol}$, $66 \mathrm{mg}$ ), $\mathrm{CuI}$ (3 mg, $10 \mathrm{~mol} \%$ ), 2-(isobutyryl)cyclohexanone (6 mg, $20 \mathrm{~mol} \%$, DMF $(1 \mathrm{ml})$ and $\mathrm{Cs}_{2} \mathrm{CO}_{3}(0.225 \mathrm{mmol}, 75 \mathrm{mg})$. The reaction mixture was stirred at $110^{\circ} \mathrm{C}$ for $24 \mathrm{hrs}$, cooled to ambient temperature, diluted with $\mathrm{CH}_{2} \mathrm{Cl}_{2}(5 \mathrm{ml})$, the residue was filtered off, washed with $\mathrm{CH}_{2} \mathrm{Cl}_{2}(5 \mathrm{ml})$, combined organic fractions were evaporated in vacuo and chromatographed on silica gel using a gradient of eluents: $\mathrm{CH}_{2} \mathrm{Cl}_{2}-\mathrm{CH}_{2} \mathrm{Cl}_{2} / \mathrm{MeOH} 3: 1$. The target compound was eluted with $\mathrm{CH}_{2} \mathrm{Cl}_{2} / \mathrm{MeOH}_{2}: 1$. Yield: $14 \mathrm{mg}$ (10\%), deep-red solid. Mass spectrum (MALDI TOF) $\mathrm{m} / \mathrm{z}: 890.4865[\mathrm{M}]^{+}$. $\mathrm{C}_{52} \mathrm{H}_{70} \mathrm{~N}_{6} \mathrm{O}_{3} \mathrm{Zn} .{ }^{1} \mathrm{H}$ NMR $\left(\mathrm{MHz}, \mathrm{CDCl}_{3}, 298 \mathrm{~K}\right) \delta_{\mathrm{H}}$ ppm: $0.96(6 \mathrm{H}$, t, $\left.{ }^{3} J=7.3 \mathrm{~Hz}\right), 1.55\left(4 \mathrm{H}\right.$, sextet, $\left.{ }^{3} \mathrm{~J}=7.1 \mathrm{~Hz}\right), 1.67-1.78(6 \mathrm{H}, \mathrm{m})$, $1.95\left(2 \mathrm{H}\right.$, quintet, $\left.{ }^{3} J=5.9 \mathrm{~Hz}\right), 2.28\left(4 \mathrm{H}\right.$, quintet, $\left.{ }^{3} \mathrm{~J}=7.2 \mathrm{~Hz}\right), 2.60$ 
(6H, s), $2.91(2 \mathrm{H}$, br.s $), 3.17$ (2H, br.s), $3.23\left(2 \mathrm{H}, \mathrm{q},{ }^{3} J=6.1 \mathrm{~Hz}\right)$, $3.38-3.64(8 \mathrm{H}, \mathrm{m}), 3.52(6 \mathrm{H}, \mathrm{s}), 3.58(6 \mathrm{H}, \mathrm{s}), 3.66(2 \mathrm{H}, \mathrm{br} . \mathrm{s}), 3.96-$ $4.04(4 \mathrm{H}, \mathrm{m}), 6.99\left(1 \mathrm{H}, \mathrm{d},{ }^{3} J=7.7 \mathrm{~Hz}\right), 7.32(1 \mathrm{H}, \mathrm{s}), 7.38(1 \mathrm{H}$, br.d, $\left.{ }^{3} J_{o b s}=6.6 \mathrm{~Hz}\right), 7.47\left(1 \mathrm{H}, \mathrm{t},{ }^{3} \mathrm{~J}=7.6 \mathrm{~Hz}\right), 9.87(1 \mathrm{H}, \mathrm{s}), 10.00(2 \mathrm{H}, \mathrm{s})$, $\mathrm{NH}$ protons were not assigned.

Porphyrin 13. A two-necked flask flushed with dry argon, equipped with a magnetic stirrer and reflux condenser was charged with porphyrin $9(0.1 \mathrm{mmol}, 96 \mathrm{mg})$, propane-1,3-diamine (3) (0.3 mmol, $22 \mathrm{mg}), \mathrm{Pd}(\mathrm{dba}){ }_{2}(2.5 \mathrm{mg}, 4 \mathrm{~mol} \%)$, BINAP (3 mg, $4.5 \mathrm{~mol} \%)$, absolute dioxane $(1 \mathrm{ml})$ and ${ }^{t} \mathrm{BuONa}(0.3 \mathrm{mmol}$, $29 \mathrm{mg}$ ). The reaction mixture was refluxed for $24 \mathrm{hrs}$, cooled to ambient temperature, diluted with $\mathrm{CH}_{2} \mathrm{Cl}_{2}(5 \mathrm{ml})$, the residue was filtered off, washed with $\mathrm{CH}_{2} \mathrm{Cl}_{2}(5 \mathrm{ml})$, combined organic fractions were evaporated in vacuo, and chromatographed on silica gel using a gradient of eluents: $\mathrm{CH}_{2} \mathrm{Cl}_{2}-\mathrm{CH}_{2} \mathrm{Cl}_{2} / \mathrm{MeOH} 3: 1-\mathrm{CH}_{2} \mathrm{Cl}_{2}$ / $\mathrm{MeOH} / \mathrm{NH}_{3}$ aq 100:20:1-10:14:1. The target compound was eluted with $\mathrm{CH}_{2} \mathrm{Cl}_{2} / \mathrm{MeOH} / \mathrm{NH}_{3}$ aq 100:20:3. Yield: $46 \mathrm{mg}$ (49 \%), deepred solid. Mass spectrum (MALDI TOF) $m / z$ : $942.7058[\mathrm{M}]^{+}$. $\mathrm{C}_{62} \mathrm{H}_{86} \mathrm{~N}_{8} .{ }^{1} \mathrm{H}$ NMR $\left(\mathrm{CDCl}_{3}, 298 \mathrm{~K}\right) \delta_{\mathrm{H}}$ ppm: -2.30 (2H, br.s), 1.00 $\left(12 \mathrm{H}, \mathrm{t},{ }^{3} \mathrm{~J}=7.3 \mathrm{~Hz}\right), 1.57\left(8 \mathrm{H}\right.$, sextet, $\left.{ }^{3} \mathrm{~J}=7.3 \mathrm{~Hz}\right), 1.77$ ( $8 \mathrm{H}$, quintet, $\left.{ }^{3} J=7.3 \mathrm{~Hz}\right), 1.88\left(4 \mathrm{H}\right.$, quintet, $\left.{ }^{3} J=6.4 \mathrm{~Hz}\right), 2.25$ ( $8 \mathrm{H}$, quintet, $\left.{ }^{3} J_{o b s}=5.8 \mathrm{~Hz}\right), 2.65(12 \mathrm{H}, \mathrm{s}), 2.93\left(4 \mathrm{H}, \mathrm{t},{ }^{3} \mathrm{~J}=6.4 \mathrm{~Hz}\right), 3.37(4 \mathrm{H}, \mathrm{t}$, $\left.{ }^{3} J=6.4 \mathrm{~Hz}\right), 4.03\left(8 \mathrm{H}, \mathrm{t},{ }^{3} J_{o b s}=5.8 \mathrm{~Hz}\right), 6.92\left(4 \mathrm{H}, \mathrm{d},{ }^{3} J_{o b s}=8.1 \mathrm{~Hz}\right)$, $7.80\left(4 \mathrm{H}, \mathrm{d},{ }^{3} \mathrm{~J}_{o b s}=8.1 \mathrm{~Hz}\right), 10.26(2 \mathrm{H}, \mathrm{s})$, six NH protons were not assigned. ${ }^{13} \mathrm{C}$ NMR (??? MHz, $\left.\mathrm{CDCl}_{3}, 298 \mathrm{~K}\right) \delta_{\mathrm{C}}$ ppm: $14.2(4 \mathrm{C})$, 14.9 (4C), 22.8 (4C), 26.7 (4C), 32.5 (6C), 33.0 (4C), 40.2 (2C), 42.4 (2C), 96.6 (2C), 111.9 (4C), 118.4 (2C), 131.1 (2C), 133.5 (4C), 136.5 (4C), 141.3 (4C), 142.8 (4C), 145.9 (4C), 148.5 (2C).

Porphyrin 15. A two-necked flask flushed with dry argon, equipped with a magnetic stirrer and reflux condenser was charged with porphyrin $11(0.05 \mathrm{mmol}, 48 \mathrm{mg})$, trioxadiamine $4(0.2 \mathrm{mmol}$, $44 \mathrm{mg}$ ), Pd(dba) (4.5 mg, $16 \mathrm{~mol} \mathrm{\% ),} \mathrm{DavePhos} \mathrm{(3.5} \mathrm{mg,}$ $18 \mathrm{~mol} \%)$, absolute dioxane $(3 \mathrm{ml})$ and ${ }^{t} \mathrm{BuONa}(0.15 \mathrm{mmol}$, $15 \mathrm{mg}$ ). The reaction mixture was refluxed for $24 \mathrm{hrs}$, cooled to ambient temperature, diluted with $\mathrm{CH}_{2} \mathrm{Cl}_{2}(5 \mathrm{ml})$, the residue was filtered off, washed with $\mathrm{CH}_{2} \mathrm{Cl}_{2}(5 \mathrm{ml})$, combined organic fractions were evaporated in vacuo, and chromatographed on silica gel using a gradient of eluents: $\mathrm{CH}_{2} \mathrm{Cl}_{2}-\mathrm{CH}_{2} \mathrm{Cl}_{2} / \mathrm{MeOH} 3: 1-\mathrm{CH}_{2} \mathrm{Cl}_{2} /$ $\mathrm{MeOH} / \mathrm{NH}_{3}$ aq 100:20:1-10:14:1. The target compound was eluted with $\mathrm{CH}_{2} \mathrm{Cl}_{2} / \mathrm{MeOH} / \mathrm{NH}_{3}$ aq 100:20:2. Yield: $9 \mathrm{mg}$ (15\%), deepred solid. Mass spectrum (MALDI TOF) $m / z$ : $1236.91[\mathrm{M}]^{+}$. $\mathrm{C}_{76} \mathrm{H}_{116} \mathrm{~N}_{8} \mathrm{O}_{6} .{ }^{1} \mathrm{H}$ NMR $\left(\mathrm{CDCl}_{3}, 298 \mathrm{~K}\right) \delta_{\mathrm{H}}$ ppm: $-2.45(2 \mathrm{H}$, br.s $)$, $0.95\left(12 \mathrm{H}, \mathrm{t},{ }^{3} J=7.1 \mathrm{~Hz}\right), 1.31$ (4H, quintet, $\left.{ }^{3} J=5.8 \mathrm{~Hz}\right), 1.53(8 \mathrm{H}$, sextet, $\left.{ }^{3} J=7.2 \mathrm{~Hz}\right), 1.72\left(8 \mathrm{H}\right.$, quintet, $\left.{ }^{3} J=7.2 \mathrm{~Hz}\right), 1.96$ (4H, quintet, $\left.{ }^{3} J=6.2 \mathrm{~Hz}\right), 2.19\left(8 \mathrm{H}\right.$, quintet, $\left.{ }^{3} J=7.2 \mathrm{~Hz}\right), 2.66(12 \mathrm{H}, \mathrm{s}), 2.88(4 \mathrm{H}$, $\left.\mathrm{t},{ }^{3} \mathrm{~J}=6.2 \mathrm{~Hz}\right), 3.03\left(4 \mathrm{H}, \mathrm{t},{ }^{3} \mathrm{~J}=6.3 \mathrm{~Hz}\right), 3.16\left(4 \mathrm{H}, \mathrm{t},{ }^{3} J_{o b s}=4.9 \mathrm{~Hz}\right)$, $3.33\left(4 \mathrm{H}, \mathrm{t},{ }^{3} \mathrm{~J}_{\mathrm{obs}}=4.6 \mathrm{~Hz}\right), 3.37\left(4 \mathrm{H}, \mathrm{t},{ }^{3} \mathrm{~J}=6.3 \mathrm{~Hz}\right), 3.41-3.67(12 \mathrm{H}$, m), $3.98\left(8 \mathrm{H}, \mathrm{t},{ }^{3} J=7.0 \mathrm{~Hz}\right), 7.02\left(2 \mathrm{H}, \mathrm{d},{ }^{3} J=7.7 \mathrm{~Hz}\right), 7.27(2 \mathrm{H}, \mathrm{s})$, $7.39\left(2 \mathrm{H}\right.$, br.d, $\left.{ }^{3} J_{o b s}=6.7 \mathrm{~Hz}\right), 7.50\left(2 \mathrm{H}\right.$, br.t, $\left.{ }^{3} J_{o b s}=6.8 \mathrm{~Hz}\right), 10.21$ $(2 \mathrm{H}, \mathrm{s})$, six $\mathrm{NH}$ protons were not assigned.

Porphyrin 19 was obtained as the second product in the synthesis of porphyrin 15. Eluent $\mathrm{CH}_{2} \mathrm{Cl}_{2} / \mathrm{MeOH} 3: 1$. Yield: $7 \mathrm{mg}$ (12\%), deep-red solid. Mass spectrum (MALDI TOF) $\mathrm{m} / \mathrm{z}$ : $1096.6558[\mathrm{M}]^{+} . \mathrm{C}_{66} \mathrm{H}_{93} \mathrm{BrN}_{6} \mathrm{O}_{3} .{ }^{1} \mathrm{H}$ NMR $\left(\mathrm{CDCl}_{3}, 298 \mathrm{~K}\right) \delta_{\mathrm{H}}$ ppm: -2.45 (2H, br.s), $0.95\left(12 \mathrm{H}, \mathrm{t},{ }^{3} J=7.1 \mathrm{~Hz}\right), 1.52$ (8H, sextet, $\left.{ }^{3} J=7.2 \mathrm{~Hz}\right), 1.68-1.75(10 \mathrm{H}, \mathrm{m}), 1.90\left(2 \mathrm{H}\right.$, quintet, $\left.{ }^{3} J=6.7 \mathrm{~Hz}\right)$, $2.18\left(8 \mathrm{H}\right.$, quintet, $\left.{ }^{3} \mathrm{~J}=6.9 \mathrm{~Hz}\right), 2.64(12 \mathrm{H}, \mathrm{s}), 2.87$ (2H, br.s), 2.94 (2H, br.s), 3.14 (2H, br.s), 3.28 ( $\left.2 \mathrm{H}, \mathrm{t},{ }^{3} J=6.2 \mathrm{~Hz}\right), 3.37$ (2H, br.s), 3.56 (6H, br.s), 3.96 (8H, br.s), $7.04\left(1 \mathrm{H}, \mathrm{d},{ }^{3} J=7.8 \mathrm{~Hz}\right), 7.28(1 \mathrm{H}$, s), $7.41\left(1 \mathrm{H}, \mathrm{d},{ }^{3} J=7.1 \mathrm{~Hz}\right), 7.48\left(1 \mathrm{H}, \mathrm{t},{ }^{3} J=7.6 \mathrm{~Hz}\right), 7.60(1 \mathrm{H}, \mathrm{t}$, $\left.{ }^{3} J=7.5 \mathrm{~Hz}\right), 7.93\left(1 \mathrm{H}, \mathrm{d},{ }^{3} J=8.2 \mathrm{~Hz}\right), 8.01\left(1 \mathrm{H}, \mathrm{d},{ }^{3} J=8.1 \mathrm{~Hz}\right), 8.27$ $(1 \mathrm{H}, \mathrm{s}), 10.22(2 \mathrm{H}, \mathrm{s})$, three $\mathrm{NH}$ protons were not assigned.

Porphyrin 16. A two-necked flask flushed with dry argon, equipped with a magnetic stirrer and reflux condenser was charged with porphyrin $11(0.05 \mathrm{mmol}, 48 \mathrm{mg})$, dioxadiamine $12(0.2 \mathrm{mmol}$, $41 \mathrm{mg}), \mathrm{Pd}(\mathrm{dba})_{2}(4.5 \mathrm{mg}, 16 \mathrm{~mol} \%$ ), DavePhos (3.5 mg, $18 \mathrm{~mol} \%$ ), absolute dioxane $(3 \mathrm{ml})$ and ${ }^{t} \mathrm{BuONa}(0.15 \mathrm{mmol}, 15 \mathrm{mg})$. The reaction mixture was refluxed for $24 \mathrm{hrs,} \mathrm{cooled} \mathrm{to} \mathrm{ambient} \mathrm{temperature,}$ diluted with $\mathrm{CH}_{2} \mathrm{Cl}_{2}(5 \mathrm{ml})$, the residue was filtered off, washed with $\mathrm{CH}_{2} \mathrm{Cl}_{2}(5 \mathrm{ml})$, combined organic fractions were evaporated in vac$u o$, and chromatographed on silica gel using a gradient of eluents: $\mathrm{CH}_{2} \mathrm{Cl}_{2}-\mathrm{CH}_{2} \mathrm{Cl}_{2} / \mathrm{MeOH} 3: 1-\mathrm{CH}_{2} \mathrm{Cl}_{2} / \mathrm{MeOH} / \mathrm{NH}_{3}$ aq 100:20:110:14:1. The target compound was eluted with $\mathrm{CH}_{2} \mathrm{Cl}_{2} / \mathrm{MeOH} /$ $\mathrm{NH}_{3}$ aq 100:20:2. Yield: $18 \mathrm{mg}$ (30\%), deep-red solid. Mass spectrum (MALDI TOF) $m / z: 1204.9049[\mathrm{M}]^{+} . \mathrm{C}_{76} \mathrm{H}_{116} \mathrm{~N}_{8} \mathrm{O}_{4} .{ }^{1} \mathrm{H} \mathrm{NMR}\left(\mathrm{CDCl}_{3}\right.$, $298 \mathrm{~K}) \delta_{\mathrm{H}}$ ppm: $-2.43\left(2 \mathrm{H}\right.$, br.s), $0.97\left(12 \mathrm{H}, \mathrm{t},{ }^{3} J=7.0 \mathrm{~Hz}\right), 1.43(4 \mathrm{H}$, quintet, $\left.{ }^{3} J=6.6 \mathrm{~Hz}\right), 1.49-1.57(12 \mathrm{H}, \mathrm{m}), 1.69-1.76(12 \mathrm{H}, \mathrm{m}), 1.95$ (4H, quintet, $\left.{ }^{3} J=5.9 \mathrm{~Hz}\right), 2.21\left(8 \mathrm{H}\right.$, br.quintet, $\left.{ }^{3} \mathrm{~J}_{\text {obs }}=6.2 \mathrm{~Hz}\right), 2.53$ $\left(4 \mathrm{H}, \mathrm{t},{ }^{3} \mathrm{~J}=7.0 \mathrm{~Hz}\right), 2.68(12 \mathrm{H}, \mathrm{s}), 3.14\left(4 \mathrm{H}\right.$, br.t, $\left.{ }^{3} J_{o b s}^{o b s}=5.1 \mathrm{~Hz}\right), 3.25$ $3.45(12 \mathrm{H}, \mathrm{m}), 3.57\left(4 \mathrm{H}\right.$, br.t, $\left.{ }^{3} \mathrm{~J}_{\text {obs }}=4.6 \mathrm{~Hz}\right), 4.00\left(8 \mathrm{H}, \mathrm{t},{ }^{3} \mathrm{~J}=7.0 \mathrm{~Hz}\right)$ $7.02\left(2 \mathrm{H}, \mathrm{d},{ }^{3} \mathrm{~J}=7.1 \mathrm{~Hz}\right), 7.30(2 \mathrm{H}, \mathrm{s}), 7.43\left(2 \mathrm{H}\right.$, br.d, $\left.{ }^{3} \mathrm{~J}_{\text {obs }}=5.6 \mathrm{~Hz}\right)$, $7.51\left(2 \mathrm{H}, \mathrm{t},{ }^{3} \mathrm{~J}=7.3 \mathrm{~Hz}\right)$, six NH protons were not assigned. ${ }^{13} \mathrm{C} \mathrm{NMR}$ $\left(\mathrm{CDCl}_{3}, 298 \mathrm{~K}\right) \delta_{\mathrm{C}} \mathrm{ppm}: 14.2(4 \mathrm{C}), 14.4(4 \mathrm{C}), 22.8(4 \mathrm{C}), 26.3(2 \mathrm{C})$, 26.4 (2C), 26.7 (4C), 29.5 (2C), 32.5 (6C), 33.0 (4C), 39.4 (2C), 42.3 (2C), 68.7 (2C), 69.4 (2C), 70.5 (2C), 70.8 (2C), 96.6 (2C), 112.7 (2C), 118.1 (2C), $118.6(2 \mathrm{C}), 122.8$ (2C), 128.2 (2C), 136.4 (4C), 141.2 (4C), 143.0 (4C), 145.0 (4C), 148.0 (2C), 2 quaternary carbon atoms were not assigned.

Porphyrin 20 was obtained as the second product in the synthesis of porphyrin 16. Eluent $\mathrm{CH}_{2} \mathrm{Cl}_{2} / \mathrm{MeOH}$ 10:1-3:1. Yield: $10 \mathrm{mg}(18 \%)$, deep-red solid. Mass spectrum (MALDI TOF) $\mathrm{m} / \mathrm{z}$ : $1080.6632[\mathrm{M}]^{+} . \mathrm{C}_{66} \mathrm{H}_{93} \mathrm{BrN}_{6} \mathrm{O}_{2} .{ }^{1} \mathrm{H} \mathrm{NMR}\left(\mathrm{CDCl}_{3}, 298 \mathrm{~K}\right) \delta_{\mathrm{H}} \mathrm{ppm}$ : -2.35 (2H, br.s), $0.95\left(12 \mathrm{H}\right.$, br.t, $\left.{ }^{3} J_{o b s}=6.2 \mathrm{~Hz}\right), 1.25-1.63(20 \mathrm{H}$, br.m), 1.72 (8H, br.s), 1.90 (4H, br.s), $2.20\left(8 \mathrm{H}\right.$, br.t, $\left.{ }^{3} \mathrm{~J}_{\text {obs }}=7.0 \mathrm{~Hz}\right)$, $2.65(12 \mathrm{H}, \mathrm{s}), 2.94-3.65$ (24H, br.m), 3.97 (8H, br.s), $7.01(1 \mathrm{H}$, br.d, $\left.{ }^{3} J_{o b s}=7.0 \mathrm{~Hz}\right), 7.27(1 \mathrm{H}, \mathrm{s}), 7.40\left(1 \mathrm{H}\right.$, br.d, $\left.{ }^{3} J_{o b s}=6.1 \mathrm{~Hz}\right), 7.50$ $\left(1 \mathrm{H}\right.$, br.t, $\left.{ }^{3} J_{o b s}=7.0 \mathrm{~Hz}\right), 7.60\left(1 \mathrm{H}\right.$, br.y, $\left.{ }^{3}{ }^{o b s}=7.2 \mathrm{~Hz}\right), 7.95(1 \mathrm{H}, \mathrm{d}$, $\left.{ }^{3} J=7.5 \mathrm{~Hz}\right), 8.03\left(1 \mathrm{H}, \mathrm{d},{ }^{3} J=8.2 \mathrm{~Hz}\right), 8.28(1 \mathrm{H}, 2), 10.23(2 \mathrm{H}, \mathrm{s})$, six $\mathrm{NH}$ protons were not assigned.

Conjugate 28. A two-necked flask flushed with dry argon, equipped with a magnetic stirrer and reflux condenser was charged with $\mathrm{Zn}$ porphyrinate $27(0.15 \mathrm{mmol}, 113 \mathrm{mg})$, azacrown derivative 25 (0.15 mmol, $64 \mathrm{mg}$ ), Pd(dba) ${ }_{2}(7 \mathrm{mg}, 8 \mathrm{~mol} \%$ ), BINAP (8.5 mg, $9 \mathrm{~mol} \%)$, absolute dioxane $(2 \mathrm{ml})$ and ${ }^{t} \mathrm{BuONa}(0.23 \mathrm{mmol}$, $22 \mathrm{mg}$ ). The reaction mixture was refluxed for $24 \mathrm{hrs}$, cooled to ambient temperature, diluted with $\mathrm{CH}_{2} \mathrm{Cl}_{2}(5 \mathrm{ml})$, the residue was filtered off, washed with $\mathrm{CH}_{2} \mathrm{Cl}_{2}(5 \mathrm{ml})$, combined organic fractions were evaporated in vacuo, dissolved in $\mathrm{CH}_{2} \mathrm{Cl}_{2}$, washed with water $(5 \mathrm{ml})$ and dried over molecular sieves. Solvent was again evaporated in vacuo and the residue was chromatographed on silica gel using a gradient of eluents: $\mathrm{CH}_{2} \mathrm{Cl}_{2}-\mathrm{CH}_{2} \mathrm{Cl}_{2} / \mathrm{MeOH}$ 3:1. The target compound was eluted with $\mathrm{CH}_{2} \mathrm{Cl}_{2} / \mathrm{MeOH}$ 10:1. Yield: $24 \mathrm{mg}(15 \%)$, deep-red solid. UV-Vis $\left(\mathrm{CH}_{3} \mathrm{CN}\right) \lambda \mathrm{nm}(\lg \varepsilon)$ : 409 (5.55), 539 (4.32), 574 (4.13). Mass spectrum (MALDI TOF) $m / z: 1095.5976[\mathrm{M}]^{+} . \mathrm{C}_{64} \mathrm{H}_{85} \mathrm{~N}_{7} \mathrm{O}_{5} \mathrm{Zn} .{ }^{1} \mathrm{H}$ NMR $\left(\mathrm{CDCl}_{3}, 298 \mathrm{~K}\right) \delta_{\mathrm{H}}$ ppm: $0.97(6 \mathrm{H}, \mathrm{t}, J=7.3 \mathrm{~Hz}), 1.51$ (4H, sextet, $J=7.3 \mathrm{~Hz}), 1.69(4 \mathrm{H}$, quintet, $J=7.4 \mathrm{~Hz}$ ), 2.12 (4H, quintet, $J=7.4 \mathrm{~Hz}), 2.25$ ( $2 \mathrm{H}, \mathrm{br} . \mathrm{s})$, $2.51(6 \mathrm{H}, \mathrm{s}), 2.70-3.50(26 \mathrm{H}$, br.m $), 3.11(2 \mathrm{H}, \mathrm{br} . \mathrm{s}), 3.34(2 \mathrm{H}, \mathrm{t}$, $J=6.2 \mathrm{~Hz}), 3.41(6 \mathrm{H}, \mathrm{s}), 3.43(6 \mathrm{H}, \mathrm{s}), 3.71(4 \mathrm{H}, \mathrm{t}, J=7.4 \mathrm{~Hz}), 6.06$ (1H, br.s), $6.27(2 \mathrm{H}$, br.s $), 6.80\left(1 \mathrm{H}, \mathrm{t}, J_{o b s}=6.9 \mathrm{~Hz}\right), 6.92(1 \mathrm{H}, \mathrm{d}$, $\left.J_{o b s}=6.8 \mathrm{~Hz}\right), 7.14(1 \mathrm{H}, \mathrm{s}), 7.32\left(1 \mathrm{H}, \mathrm{d}, J_{o b s}=6.2 \mathrm{~Hz}\right), 7.44(1 \mathrm{H}, \mathrm{t}$, $J=7.6 \mathrm{~Hz}), 9.42(1 \mathrm{H}, \mathrm{s}), 9.82(2 \mathrm{H}, \mathrm{s})$, two $\mathrm{NH}$ protons were not assigned. ${ }^{13} \mathrm{C}$ NMR $\left(\mathrm{CDCl}_{3}, 298 \mathrm{~K}\right) \delta_{\mathrm{C}} \mathrm{ppm}: 11.5(2 \mathrm{C}), 12.1(2 \mathrm{C})$, 14.1 (2C), 15.1 (2C), 22.7 (2C), 26.3 (2C), 28.5 (1C), 32.4 (2C), 33.0 (2C), $41.6(1 \mathrm{C}), 41.7$ (1C), 51.9 (2C), 57.3 (1C), $68.8(2 \mathrm{C})$, 69.4 (4C), 69.6 (4C), 95.8 (1C), 96.7 (2C), 102.6 (1C), 112.5 (1C), 118.8 (1C), 123.0 (2C), 128.0 (1C), 135.6 (2C), 137.8 (2C), 138.4 (2C), 140.9 (2C), 144.4 (1C), 146.8 (2C), 146.9 (2C), 147.5 (2C), 147.7 (2C), 149.1 (1C), 149.2 (1C), four quaternary carbon atoms were not assigned.

Conjugate 29. A two-necked flask flushed with dry argon, equipped with a magnetic stirrer and reflux condenser was charged with $\mathrm{Zn}$ porphyrinate $\mathbf{1 0}(0.11 \mathrm{mmol}, 111 \mathrm{mg})$, azacrown derivative 25 (0.22 mmol, $94 \mathrm{mg}), \mathrm{Pd}(\mathrm{dba})_{2}(5 \mathrm{mg}, 8 \mathrm{~mol} \%)$, BINAP (6 mg, $9 \mathrm{~mol} \%)$, absolute dioxane $(2 \mathrm{ml})$ and ${ }^{\mathrm{t}} \mathrm{BuONa}(0.33 \mathrm{mmol}$, $32 \mathrm{mg}$ ). The reaction mixture was refluxed for $24 \mathrm{hrs}$ and then 
worked up in a similar manner as described for compound 28. The target compound was eluted with $\mathrm{CH}_{2} \mathrm{Cl}_{2} / \mathrm{MeOH} / \mathrm{NH}_{3}$ aq 100:35:610:4:1. Yield: $28 \mathrm{mg}$ (15\%), deep-red solid. UV-Vis $\left(\mathrm{CH}_{3} \mathrm{CN}\right) \lambda \mathrm{nm}$ $(\lg \varepsilon)$ : 417 (4.80), 546 (3.45), 578 (3.17). Mass spectrum (MALDI TOF) $m / z: 1707.03[\mathrm{M}]^{+} . \mathrm{C}_{100} \mathrm{H}_{142} \mathrm{~N}_{10} \mathrm{O}_{10} \mathrm{Zn} .{ }^{1} \mathrm{H}$ NMR $\left(\mathrm{CDCl}_{3}, 298\right.$ K) $\delta_{\mathrm{H}}$ ppm: $0.96(12 \mathrm{H}, \mathrm{t}, J=7.3 \mathrm{~Hz}), 1.53(8 \mathrm{H}$, sextet, $J=7.0 \mathrm{~Hz})$, $1.73(8 \mathrm{H}$, quintet, $J=6.8 \mathrm{~Hz}), 2.01$ (4H, br.s), 2.18 (8H, quintet, $J=6.4 \mathrm{~Hz}), 2.56(12 \mathrm{H}, \mathrm{s}), 2.70$ ( $8 \mathrm{H}$, br.s $), 3.21$ (4H, br.s), 3.35$3.68(48 \mathrm{H}, \mathrm{m}), 3.94(8 \mathrm{H}, \mathrm{t}, J=7.1 \mathrm{~Hz}), 6.44\left(2 \mathrm{H}, \mathrm{d}, J_{o b s}=5.4 \mathrm{~Hz}\right)$, $6.63\left(4 \mathrm{H}, \mathrm{d}, J_{o b s}=7.0 \mathrm{~Hz}\right), 6.93\left(4 \mathrm{H}, \mathrm{d}, J_{o b s}=7.8 \mathrm{~Hz}\right), 7.10(2 \mathrm{H}$, $\mathrm{t}, J=7.8 \mathrm{~Hz}), 7.77\left(4 \mathrm{H}, \mathrm{d}, J_{o b s}=7.8 \mathrm{~Hz}\right), 10.10(2 \mathrm{H}, \mathrm{s})$, four $\mathrm{NH}$ protons were not assigned. $14.2(4 \mathrm{C}), 15.5$ (4C), $22.8(4 \mathrm{C}), 26.7$ (4C), 29.6 (2C), 32.5 (4C), 33.1 (4C), 42.0 (2C), 42.2 (2C), 53.6 (4C), 59.3 (2C), 69.7-70.6 (m, 20C), 97.0 (2C), 111.7 (2C), 112.0 (4C), 114.3 (2C), 118.4 (2C), 128.7 (2C), 129.0 (4C), 133.7 (4C), 138.2 (4C), 142.8 (4C), 146.2 (4C), 148.2 (2C), 148.4 (4C), four quaternary carbon atoms were not assigned.

Conjugate 30. A two-necked flask flushed with dry argon, equipped with a magnetic stirrer and reflux condenser was charged with porphyrin $9(0.1 \mathrm{mmol}, 96 \mathrm{mg})$, azacrown derivative 26 (0.2 mmol, $76 \mathrm{mg}), \mathrm{Pd}(\mathrm{dba})_{2}(9 \mathrm{mg}, 16 \mathrm{~mol} \%)$, BINAP (11 mg, $18 \mathrm{~mol} \%)$, absolute dioxane $(1 \mathrm{ml})$ and ${ }^{t} \mathrm{BuONa}(0.3 \mathrm{mmol}, 29 \mathrm{mg})$. The reaction mixture was refluxed for $24 \mathrm{hrs}$ and then worked up in a similar manner as described for compound 28. The target compound was eluted with $\mathrm{CH}_{2} \mathrm{Cl}_{2} / \mathrm{MeOH} / \mathrm{NH}_{3}$ aq 100:20:3. Yield: $32 \mathrm{mg}$ (20\%), deep-red solid. Mass spectrum (MALDI TOF) $\mathrm{m} / \mathrm{z}$ : $1557.04[\mathrm{M}]^{+} . \mathrm{C}_{96} \mathrm{H}_{136} \mathrm{~N}_{10} \mathrm{O}_{8} .{ }^{1} \mathrm{H}$ NMR $\left(\mathrm{CDCl}_{3}, 298 \mathrm{~K}\right) \delta_{\mathrm{H}}$ ppm: -2.40 $(2 \mathrm{H}$, br.s) $0.95(12 \mathrm{H}, \mathrm{t}, J=7.2 \mathrm{~Hz}), 1.51(8 \mathrm{H}$, sextet, $J=7.3 \mathrm{~Hz})$, $1.71(8 \mathrm{H}$, quintet, $J=7.4 \mathrm{~Hz}), 2.15(4 \mathrm{H}$, quintet, $J=5.8 \mathrm{~Hz}), 2.19$ $(8 \mathrm{H}$, quintet, $J=7.1 \mathrm{~Hz}), 2.61(12 \mathrm{H}, \mathrm{s}), 2.84(8 \mathrm{H}, \mathrm{br} . \mathrm{s}), 3.42(4 \mathrm{H}$, t, $J=6.3 \mathrm{~Hz}), 3.53(4 \mathrm{H}, \mathrm{t}, J=6.1 \mathrm{~Hz}), 3.64(36 \mathrm{H}, \mathrm{br} . \mathrm{s}), 3.98(8 \mathrm{H}$, $\mathrm{t}, J=7.0 \mathrm{~Hz}), 6.60(2 \mathrm{H}, \mathrm{d}, J=7.6 \mathrm{~Hz}), 6.69\left(2 \mathrm{H}, \mathrm{d}, J_{o b s}=7.1 \mathrm{~Hz}\right)$, $6.86(2 \mathrm{H}, \mathrm{br} . \mathrm{s}), 6.98\left(4 \mathrm{H}, \mathrm{d}, J_{\text {obs }}=8.1 \mathrm{~Hz}\right), 7.17(2 \mathrm{H}, \mathrm{t}, J=7.7 \mathrm{~Hz})$, $7.79\left(4 \mathrm{H}, \mathrm{d}, J_{o b s}=8.1 \mathrm{~Hz}\right), 10.20(2 \mathrm{H}, \mathrm{s})$, four NH protons were not assigned. ${ }^{13} \mathrm{C}$ NMR $\left(\mathrm{CDCl}_{3}, 298 \mathrm{~K}\right) \delta_{\mathrm{C}} \mathrm{ppm}: 14.2(4 \mathrm{C}), 14.9$ (4C), 22.8 (4C), 26.7 (4C), 29.5 (2C), 32.5 (4C), 33.0 (4C), 42.2 (2C), 42.4 (2C), 54.4 (4C), 60.7 (2C), 69.9 (4C), 70.2 (4C), 70.5 (4C), 70.9 (4C), 96.6 (2C), 111.1 (2C), 112.0 (4C), 113.7 (2C), 118.1 (4C), 122.5 (2C), 129.1 (2C), 133.5 (4C), 136.5 (4C), 141.3 (4C), 142.8 (4C), 145.8 (2C), 148.3 (2C), 148.4 (2C), four quaternary carbon atoms were not assigned.

\section{Results and Discussion}

In the course of our investigation we proposed several approaches to create a combination of the porphyrin moiety with azacrown ethers. The first route employed the diamine or oxadiamine linker between these two macrocycles, and for this purpose first the amination reactions of meso(halogenophenyl)porphyrins $\mathbf{1}$ and $\mathbf{2}$ with propane-1,3-diamine (3) and trioxadiamine $\mathbf{4}$ were studied. Amination of meso(4-bromophenyl)porphyrin $\mathbf{1}$ taken as its $\mathrm{Zn}$ complex was conducted using a standard Pd-catalyzed procedure in the presence of $\mathrm{Pd}(\mathrm{dba})_{2} / \mathrm{BINAP}$ system $(\mathrm{dba}=$ dibenzylideneacetone, $\mathrm{BINAP}=2,2$ '- bis(diphenylphosphino)-1,1'binaphthalene). The reaction was carried out using 3 equiv. of the trioxadiamine 4 in boiling dioxane with ${ }^{t} \mathrm{BuONa}$ as a base and provided $16 \%$ yield of the target derivative 5 (Scheme 1). It should be noted that the same reaction with free porphyrin was unsuccessful and did not provide the desired coupling product. It is of undoubtful interest that the $\mathrm{Cu}^{\mathrm{I}}$-catalyzed amination of 3-iodophenyl substituted $\mathrm{Zn}$ porphyrinate 2 with excess of propane-1,3-diamine (3) was substantially more successful as it provided ca $50 \%$ yield of the amination product 6. 2-(iso-Butyryl)cyclohexanone ligand $(\mathbf{L})$ was used in the reaction run in $\mathrm{DMF}$ at $110^{\circ} \mathrm{C}$. The amination of the same prophyrinate $\mathbf{2}$ with trioxadiamine 4 carried out under similar conditions was less efficient and compound 7 was isolated in $10 \%$ yield (Scheme 1). Having obtained such encouraging results, we have explored the possibility to synthesize a bisporphyrin compound with trioxadiamine linker between two porphyrin moieties and reacted 2 equiv. of $\mathbf{2}$ with trioxadiamine $\mathbf{4}$ under the catalysis by $\mathrm{CuI} / \mathbf{L} / \mathrm{DMF}$ at $140{ }^{\circ} \mathrm{C}$. However, in this case we have managed to isolate the target compound $\mathbf{8}$ in trace amounts and it could not be purified from various admixtures. It was detected by MALDI-TOF mass spectrometry $(\mathrm{m} / \mathrm{z}=1560.60$ $\left.[\mathrm{M}]^{+}\right)$. This result demonstrates, as enough general rule, that $N, N$ '-diarylation of diamines and oxadiamines is often

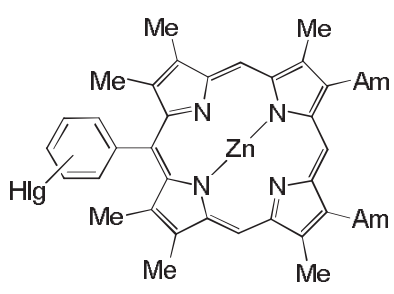

$\mathrm{Hlg}=p-\mathrm{Br}(1), m-\mathrm{I}(2)$

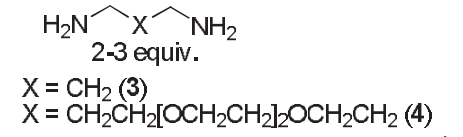

a) $\mathrm{Hlg}=\mathrm{Br} \begin{aligned} & \mathrm{Pd}(\mathrm{dba})_{2} / \mathrm{BINAP} \\ & \text { tBuONa, dioxane }\end{aligned}$

b) $\mathrm{Hlg}=\mathrm{I} \quad \mathrm{Cul} / \mathrm{L} / \mathrm{DMF}, \mathrm{Cs}_{2} \mathrm{CO}_{3}$<smiles>CC(C)C(=O)C1CCCCC1=O</smiles>

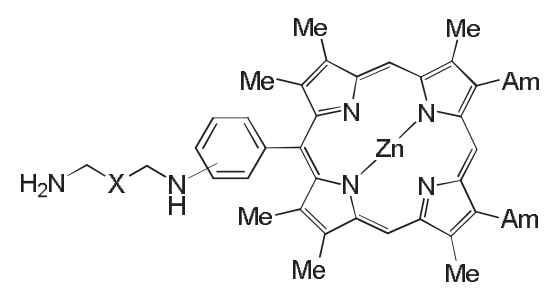

5: $p-\mathrm{NH}, \mathrm{X}=\mathrm{CH}_{2} \mathrm{CH}_{2}\left[\mathrm{CCH}_{2} \mathrm{CH}_{2}\right]_{2} \mathrm{OCH}_{2} \mathrm{CH}_{2}, 16 \%$ (via Pd catalysis) 6: $m-\mathrm{NH}, \mathrm{X}=\mathrm{CH}_{2}, 50 \%$ (via Cu catalysis)

7: $m-\mathrm{NH}, \mathrm{X}=\mathrm{CH}_{2} \mathrm{CH}_{2}\left[\mathrm{OCH}_{2} \mathrm{CH}_{2}\right]_{2} \mathrm{OCH}_{2} \mathrm{CH}_{2}, 10 \%$ (via Cu catalysis)
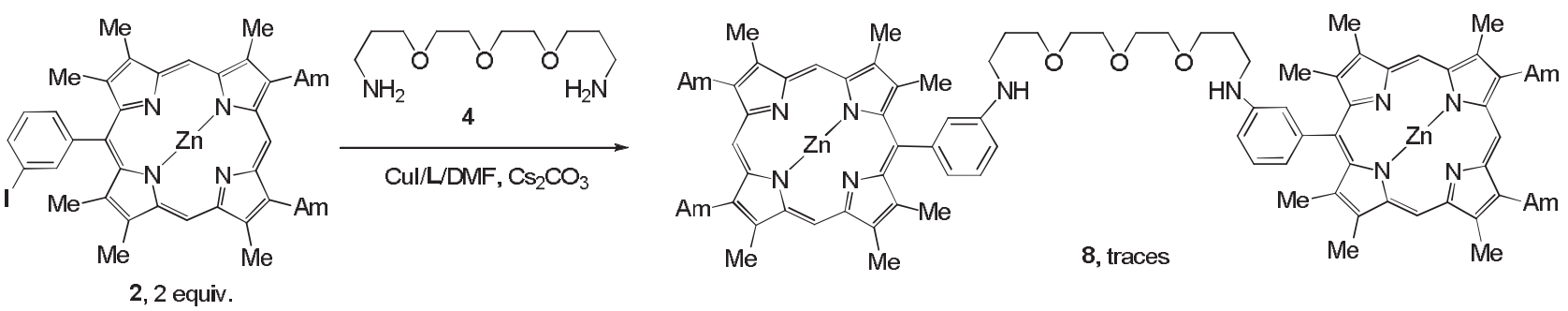

Scheme 1. 
problematic under $\mathrm{Cu}^{\mathrm{I}}$ catalysis, ${ }^{[34,35]}$ what is unlikely for $\mathrm{Pd}^{0}$-catalyzed diarylation. ${ }^{[36,37]}$

Contrary to the amination of 4-bromophenylporphyrin (1), the $\mathrm{Pd}^{0}$-catalyzed reaction of the free di-meso-(4-bromophenyl)porphyrin (9) with excess of propane-1,3-diamine was quite successful and provided $49 \%$ yield of the diamination product 13 (Scheme 2$)$. The attempt to use $\mathrm{Cu}^{\mathrm{I}}$ catalysis by reacting zinc di-meso-(4-bromophenyl)porphyrinate 10 with the same amine was unsuccessful as it has given the target diamination product $\mathbf{1 4}$ and the product of monoamination 18 only in small amounts in the reaction mixture and they could not be separated from the starting porphyrin $\mathbf{1 0}$ by the column chromatography. Compounds $\mathbf{1 4}$ and $\mathbf{1 8}$ were detected by MALDI-TOF spectra $(\mathrm{m} / \mathrm{z}=1005.64$ and $1011.44[\mathrm{M}+\mathrm{H}]^{+}$, resp.). Free porphyrin 11 with 3-bromo- phenyl substituent have reacted with excess of trioxadiamine 4 under $\mathrm{Pd}^{0}$ catalysis conditions and have given the corresponding diamination product $\mathbf{1 5}$ in $15 \%$ after column chromatography. Also the monoamination product 19 was isolated in $12 \%$ yield. A similar reaction with dioxadiamine 12 provided bis(diamino) derivative $\mathbf{1 6}$ in a better $30 \%$ yield due to easier chromatographic isolation of a less polar compound. The yield of the product of monoamination 20 was $18 \%$. These reactions were conducted in the presence of another ligand DavePhos (2-dimethylamino-2'-dicyclohexylphosphino)biphenyl which promotes diamination of dihalogenoaryl derivatives ( $m$-bromophenyl porphyrin derivative is less active in the catalytic amination than $p$-bromophenyl derivative). The reaction of $\mathrm{Zn}$ porphyrinate $\mathbf{1 2}$ with two 4-bromophenyl substituents in the porphyrin core catalyzed

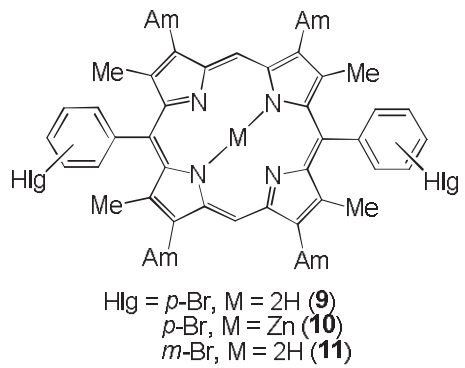

Monoamination products:

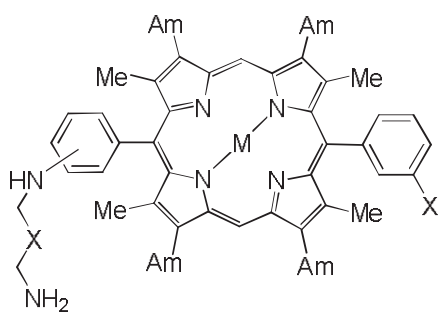

$$
\mathrm{H}_{2} \mathrm{~N} \widehat{\text { 3-6 equiv. }} \widehat{\widehat{N}} \widehat{\mathrm{NH}_{2}}
$$

$\mathrm{X}=\mathrm{CH}_{2}(\mathbf{3})$

$\mathrm{X}=\mathrm{CH}_{2} \mathrm{CH}_{2}\left[\mathrm{OCH}_{2} \mathrm{CH}_{2}\right]_{2} \mathrm{OCH}_{2} \mathrm{CH}_{2}(4)$

a) $\mathrm{Hlg}=\mathrm{Br} \quad \mathrm{Fd}(\mathrm{dba})_{2} / \mathrm{BINAP}$ or DavePhos

b) $\mathrm{HIg}=\mathrm{I} \quad \mathrm{Cul} / \mathrm{L} / \mathrm{CNF}, \mathrm{Cs}_{2} \mathrm{CO}_{3}$

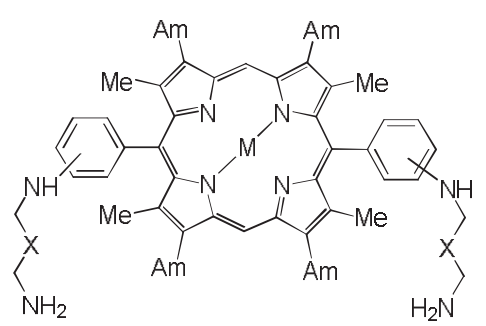

13: $p-\mathrm{NH}, \mathrm{N}=2 \mathrm{H}, \mathrm{X}=\mathrm{CH}_{2}, 4 \% \%$ (via Pd catalysis)

14: $p-\mathrm{NH}, \mathrm{N}=\mathrm{Zn}, \mathrm{X}=\mathrm{CH}_{2}$, observed in the reaction mixture (via Cu catalysis) 15: $m_{1}-\mathrm{NH}, \mathrm{M}=2 \mathrm{H}, \mathrm{X}=\mathrm{CH}_{2} \mathrm{CH}_{2}\left[\mathrm{CCH}_{2} \mathrm{Cl}_{2}\right]_{2} \mathrm{OCH}_{2} \mathrm{CH}_{2}, 15 \%$ (via Pd catalysis) 16: $m_{1}-\mathrm{NH}, \mathrm{M}=2 \mathrm{H}, \mathrm{X}=\mathrm{CH}_{2} \mathrm{CH}_{2} \mathrm{C}\left(\mathrm{Cl}_{2}\right)_{4} \mathrm{CCH}_{2} \mathrm{CH}_{2} 3 \mathrm{C} \%$ (via Pd catalysis) 17: $m-\mathrm{NH}, \mathrm{M}=\mathrm{Zn}, \mathrm{X}=\mathrm{CH}_{2} \mathrm{CH}_{2}\left[\mathrm{OCH}_{2} \mathrm{CH}_{2}\right]_{2} \mathrm{OCF}_{2} \mathrm{CH}_{2}$, traces (via Cu catalysis)

18. $p-N H, X=B r, N=Z n, X=\mathrm{CH}_{2}$, observed in the reaction mixture (via Cu catalysis)

19. $m-\mathrm{NH}, \mathrm{X}=\mathrm{Br}, \mathrm{N}=2 \mathrm{H}, \mathrm{X}=\mathrm{CH}_{2} \mathrm{CH}_{2}\left[\mathrm{OCH}_{2} \mathrm{CH}_{2}\right]_{2} \mathrm{CCH}_{2} \mathrm{CH}_{2}, 12 \%$ (via Pd catalysis)

20: $m-\mathrm{NH}, \mathrm{X}=\mathrm{Br}, \mathrm{N}=2 \mathrm{H}, \mathrm{X}=\mathrm{CH}_{2} \mathrm{CH}_{2} \mathrm{O}\left(\mathrm{CH}_{2}\right)_{4} \mathrm{CCH}_{2} \mathrm{CH}_{2}, 18 \%$

21+22: $m-\mathrm{NH}, \mathrm{X}=\mathrm{H}, \mathrm{Br}, \mathrm{N}=\mathrm{Zn}, \mathrm{X}=\mathrm{CH}_{2} \mathrm{CH}_{2}\left[\mathrm{CCH}_{2} \mathrm{CH}_{2}\right]_{2} \mathrm{OCH}_{2} \mathrm{CH}_{2}, 7 \%$ (via Cu catalysis, yield of the mixture)

\section{Scheme 2.}

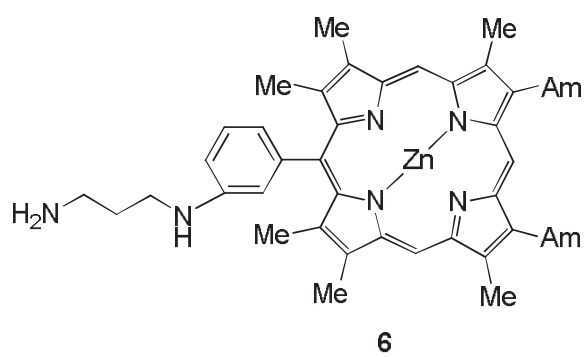<smiles>Brc1cccc(CN2CCOCCOCCOCCOCC2)c1</smiles>

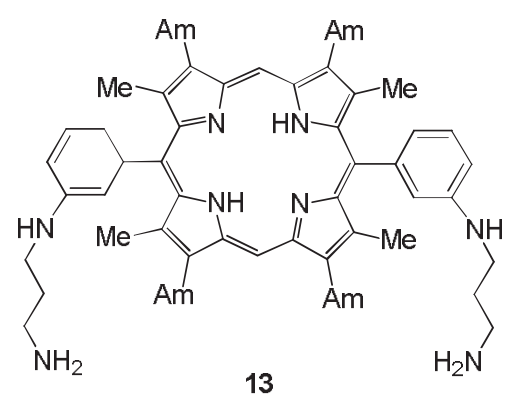

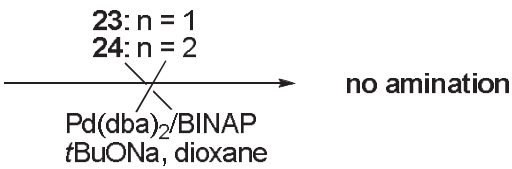

Figure 1. Unsuccessful attempts of porphyrin - azacrown ethers conjugation. 

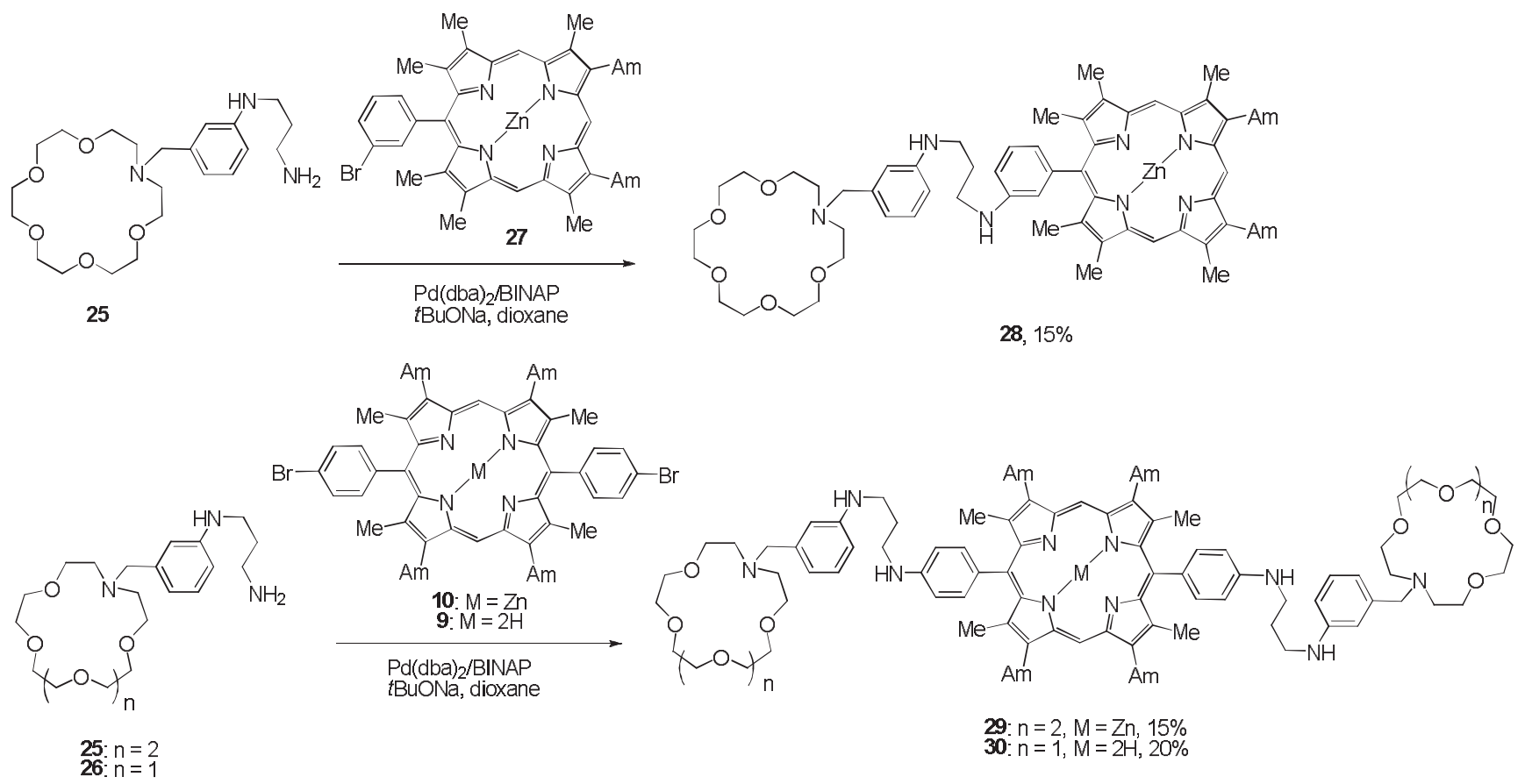

Scheme 3.

by $\mathrm{CuI} / \mathbf{L} / \mathrm{DMF}$ was inefficient and led to the products of monoamination $\mathbf{2 1}$ and $\mathbf{2 2}$ (in the latter compound the resting $\mathrm{C}-\mathrm{Br}$ bond was reduced) obtained as an inseparable mixture in ca $7 \%$ yield $\left(\mathrm{m} / \mathrm{z}=1078.49\right.$ and $1156.39[\mathrm{M}]^{+}$, resp. $)$, while the target diamination product $\mathbf{1 8}$ was isolated in trace amounts and detected using MALDI-TOF mass-spectrometry $\left(m / z=1296.79[\mathrm{M}]^{+}\right)$.

In order to obtain conjugates of azacrown ethers with porphyrins we first tried the $\mathrm{Pd}^{0}$-catalyzed coupling of mono- and diaminosubstituted porphyrins 6 and 13 with $N$-(3-bromobenzyl) substituted azacrown ethers 23 and 24 (Figure 1). However, in all attempts we did not observe the formation of the amination products even in the reaction mixtures and noted the reduction of the $\mathrm{C}-\mathrm{Br}$ bond in azacrown derivatives. Thus this way of constructing conjugates was abandoned.

Another approach for constructing azacrown-porphyrin conjugates was more successful (Scheme 3). At first amino derivatives of $N$-benzyl substituted crown ethers 25 and 26 were synthesized by the $\mathrm{Pd}^{0}$-catalyzed amination of 3-bromobenzyl substituted precursors according to a previously described procedure. ${ }^{[33]}$ Then compounds 25 and 26 were introduced in the second catalytic amination reactions with $\mathrm{Zn}$ 3-bromophenylporphyrinate 27 and with di-meso-(4-bromophenyl)porphyrins 9 and $\mathbf{1 0}$ (free base and $\mathrm{Zn}$ complex, resp.). All reactions were enough successful and provided bismacrocycle $\mathbf{2 8}$ or trismacrocycles $\mathbf{2 9 ,} \mathbf{3 0}$ in comparable 15-20\% yields (Scheme 3 ). Again it was shown that monophenyl substituted porphyrin could react normally only as its Zn complex 1 while diphenyl derivative provided similar yields of the diamination products both as free base 9 and in the form of the $\mathrm{Zn}$ complex $\mathbf{1 0}$.

Compounds 28-30 can be viewed as di- or tritopic ligands interesting for the synthesis of heterodi- or heterotrinuclear complexes. In these polymacrocycles the trimeth- ylene diamine linker between porphyrin and azacrown moieties could have been replaced for a longer trioxadiamine fragment which contains several donor atoms. This could have led to the synthesis of pentatopic ligands, unfortunately, all our attempts to change trimethylenediamine linker for trioxadiamine failed. Trioxadiamine derivatives of either meso-phenylporphyrin or $N$-benzyl azacrown ether would not participate in the $\mathrm{Pd}^{0}$-catalyzed coupling reactions similar to those shown in Figure 1 and Scheme 3. They led only to partial reduction of $\mathrm{C}-\mathrm{Br}$ bonds and formation of complex mixtures of unidentified compounds indicating severe limitations of the process.

The UV-Vis spectrum of conjugate $\mathbf{2 8}$ contains a strong Soret band $(\lambda=409 \mathrm{~nm}, \lg \varepsilon=5.55)$ and two less intensive Q bands ( $\lambda=539$ and $574 \mathrm{~nm}, \lg \varepsilon=4.32$ and 4.13 , respectively). The comparison with the starting zinc porphyrinate $\mathbf{1}$ shows insignificant bathochromic shifts of all bands by 2-3 nm and a notable increase of the intensity of Q bands. The fluorescence spectrum of compound $\mathbf{2 8}$ possesses two bands of almost equal intensities at 589 and $641 \mathrm{~nm}$. The UV-Vis spectrum of conjugate 29 contains a strong Soret band $(\lambda=417 \mathrm{~nm}, \lg \varepsilon=4.80)$ and two less intensive $Q$ bands ( $\lambda=546$ and $578 \mathrm{~nm}, \lg \varepsilon=3.45$ and 3.17 , respectively). The comparison with the starting zinc porphyrinate $\mathbf{1 0}$ shows small bathochromic shifts of all bands by $3-6 \mathrm{~nm}$ and a small increase of the intensity of the Soret band and one Q band. The fluorescence spectrum of compound 29 possesses two bands at 594 and $648 \mathrm{~nm}$, the first being more intensive. It is worthnoting that the absorption of monophenyl substituted porphyrin $\mathbf{2 8}$ in the visible range is substantially stronger than that of di-meso-phenyl substituted porphyrin 29.

We have investigated the dependence of the spectral properties of the compounds $\mathbf{2 8}$ and $\mathbf{2 9}$ possessing one and two 1-aza-18-crown-6 moieties in their structures on the presence of seventeen metal cations in order to find perspective 


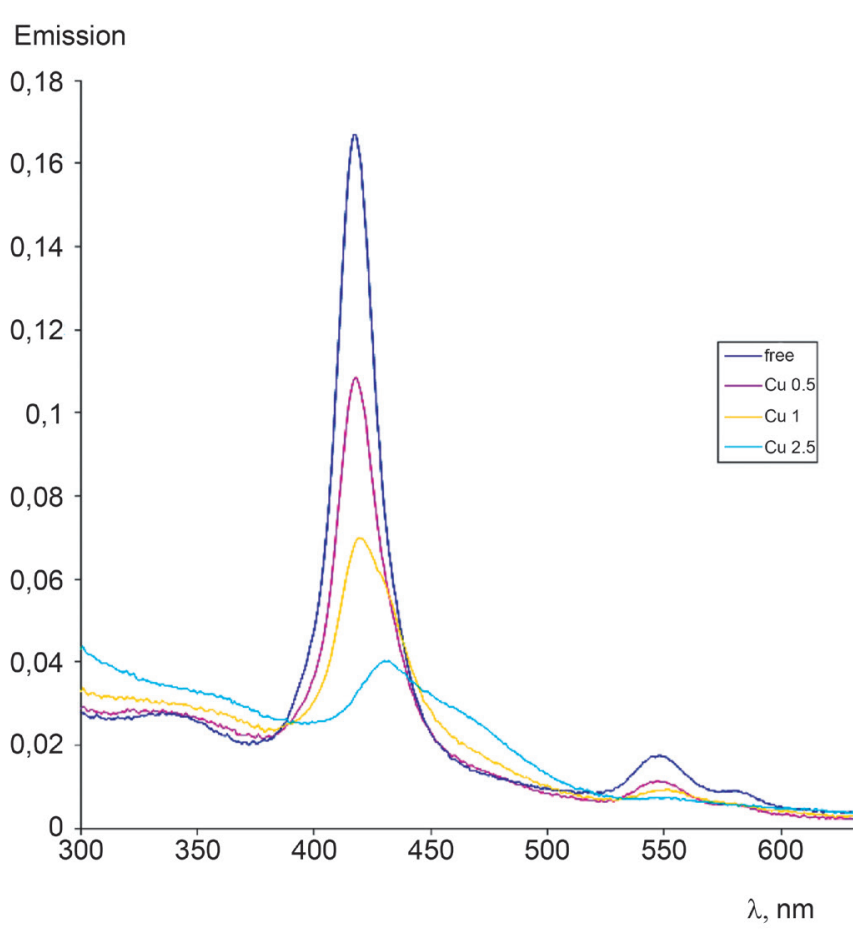

Figure 2. Evolution of UV-vis spectrum of trismacrocycle 29 after addition of $0.5,1$ and 2.5 equiv. $\mathrm{Cu}\left(\mathrm{ClO}_{4}\right)_{2}$ $\left(\mathrm{MeCN}, C=3.33 \cdot 10^{-6} \mathrm{M}\right.$ ).

optical chemosensors. For this purpose we have measured UV-Vis and fluorescent spectra of these polymacrocycles in MeCN adding 0.5, 1 and 2.5 equiv. of corresponding metal perchlorates. Excitation of bismacrocycle $\mathbf{2 8}$ was carried out at $410 \mathrm{~nm}$, that of trismacrocycle 29 - at $418 \mathrm{~nm}$. As for bismacrocycle 28, no significant changes in its electronic and fluorescent spectra could be noted for the majority of metals except for $\mathrm{Cu}^{\mathrm{II}}$ which led to a partial decrease of the intensity of the absorption peak at $409 \mathrm{~nm}$ (Figure 1S) and produced two-fold quenching of the emission intensity (Figure $2 \mathrm{~S}$ ). $\mathrm{Pb}^{\mathrm{II}}$ has also caused notable quenching of fluorescence, other metals have changed it insignificantly. The maxima of absorption and emission bands did not change in all cases studied. We have investigated the dependence of the electronic and fluorescent spectra of bismacrocycle $\mathbf{2 8}$ on the amount of copper perchlorate added. In the UV-Vis spectra one can observe gradual weakening of the absorption maximum at $409 \mathrm{~nm}$ up to 10 equiv. of $\mathrm{Cu}^{\mathrm{II}}$ added, its further attenuation together with bathochromic shift to $425 \mathrm{~nm}$ occurred in the presence of 20-30 equiv. of $\mathrm{Cu}^{\mathrm{II}}$ salt (Figure 3S). Fluorescence was quenched gradually without any shift of two maxima at 589 and $641 \mathrm{~nm}$, and with 30 equiv. of $\mathrm{Cu}\left(\mathrm{ClO}_{4}\right)_{2}$ it was almost fully quenched (Figure 4S).

The addition of 2.5 equiv. of different metal perchlorates to the solution of trismacrocycle 29 did not result in significant changes in its absorption and emission spectra (Figures $5 \mathrm{~S}$ and $6 \mathrm{~S})$, except for $\mathrm{Cu}\left(\mathrm{ClO}_{4}\right)_{2}$ which dramatically decreased the intensity of absorption and led to a bathochromic shift of its maximum (from 417 to $432 \mathrm{~nm}$, Figure 3S). The quenching of emission with $\mathrm{Cu}^{\mathrm{II}}$ was almost total (Figure $6 \mathrm{~S}$ ) while $\mathrm{Pb}^{\mathrm{II}}$ has decreased it only by $25 \%$, and the ligand was quite insensitive for other metal cations. It is interesting that the addition

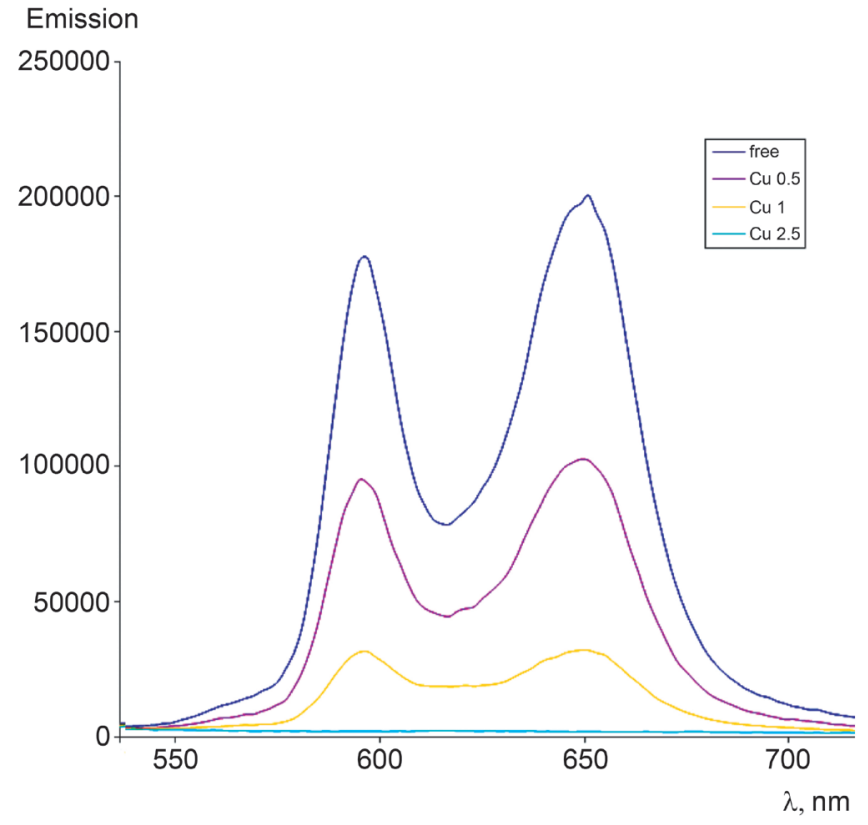

Figure 3. Evolution of the fluorescence of trismacrocycle 29 after addition of $0.5,1$ and 2.5 equiv. $\mathrm{Cu}\left(\mathrm{ClO}_{4}\right)_{2}$ $\left(\mathrm{MeCN}, C=3.33 \cdot 10^{-6} \mathrm{M}, \lambda_{e x}=418 \mathrm{~nm}\right)$.

of 0.5 and 1 equiv. of $\mathrm{Cu}^{\mathrm{II}}$ gradually decreased the absorption (Figure 2), however, it did not result in the shift of the absorption maximum both of Soret and $\mathrm{Q}$ bands. The addition of the second equivalent has given rise to above mentioned bathochromic shift of the Soret band and simultaneously the $\mathrm{Q}$ band almost totally disappeared. Also, upon the addition of 0.5 and 1 equiv. of $\mathrm{Cu}^{\mathrm{II}}$ emission gradually decreased ( $c a 2$ and 6 times, resp.), but with the second equivalent it was fully quenched (Figure 3). These facts might be explained by the formation of a non-emissive dicopper complex with trismacrocycle 29 possessing two azacrown fragments each capable of complexing with one $\mathrm{Cu}^{\mathrm{II}}$ cation.

Special experiments have been undertaken to verify cross-selectivity of the ligand 29 towards $\mathrm{Cu}^{\mathrm{II}}$ using $\mathrm{Na}^{\mathrm{I}}, \mathrm{K}^{\mathrm{I}}$, $\mathrm{Mg}^{\mathrm{II}}, \mathrm{Ca}^{\mathrm{II}}, \mathrm{Ba}^{\mathrm{II}}, \mathrm{Zn}^{\mathrm{II}}, \mathrm{Pb}^{\mathrm{II}}$ and $\mathrm{Hg}^{\mathrm{II}}$ perchlorates which can be viewed as the most expected interfering ions. The UV-Vis and fluorescence spectra in all cases were absolutely the same as in the presence of sole copper perchlorate (weak maximum at $432 \mathrm{~nm}$ and no emission). We have evaluated the detection limits of $\mathrm{Cu}^{\mathrm{II}}$ ions by the ligand 29 and found out that it was $1.2 \mathrm{mM}$ by UV spectrophotometer and $0.23 \mathrm{mM}$ by spectrofluorimeter. These figures are among the best judging by the analysis of the literature data. Indeed, there are several recently developed fluorimetric chemosensors for copper which provided detection at $1-10 \mathrm{mM}$ level, ${ }^{[38-42]}$ while there is only one sensor based on thiacalix[4]crown ether with two pyrene fluorophores able to detect $40 \mathrm{~nm}$ concentration of $\mathrm{Cu}^{\mathrm{II}} \cdot{ }^{[43]}$ It should be also noted that there is a plenty of colorimetric detectors of copper ions, ${ }^{[44-51]}$ however, their detection limits are not as low, moreover, some of them are indeed not chemosensors but rather molecular probes as they sense also other cations like $\mathrm{Hg}^{\mathrm{II}}$ or $\mathrm{Fe}^{\mathrm{III}}$. Thus conjugate 29 can be proposed as a perspective two-channel fluorimetric and colorimetric chemosensor for $\mathrm{Cu}^{\mathrm{II}}$ ions. 


\section{Conclusions}

To sum up, we have showed the possibility to decorate porphyrins with diamine and oxadiamine podands via $\mathrm{Pd}^{0}$ and $\mathrm{Cu}^{\mathrm{I}}$-catalyzed amination reactions, however, the palladium catalysis proved to be more universal. $\mathrm{Pd}^{0}-$ catalyzed amination was applied to the synthesis of bis- and trismacrocyclic conjugates of porphyrin with azacrown ethers using trimethylenediamine linkers. This target can be achieved using only one approach, i.e. combining meso(bromophenyl) substituted porphyrins with aminobenzyl derivatives of azacrown ethers. All these compounds can be used as polytopic ligands in the synthesis of polynuclear metal complexes. The properties of two compounds, bismacrocycle $\mathbf{2 8}$ and trismacrocycle $\mathbf{2 9}$, to perform as colorimetric and fluorescent sensors for metal cations were investigated, and ligand 29 possessing two 1-aza18-crown- 6 moieties arranged symmetrically around the porphyrin core was found to be enough selective for $\mathrm{Cu}^{\mathrm{II}}$ in the presence of other metals by strong emission quenching caused by copper ions.

Acknowledgements. This work was financially supported by the RFBR grant 13-03-00813.

\section{References}

1. Beletskaya I.P., Tyurin V.S., Uglov A., Stern C., Guilard R. Survey of Synthetic Routes for Synthesis and Substitution in Porphyrins. In: Handbook of Porphyrin Science, Vol. 23, 2012. p. 83.

2. Gao G.Y., Chen Y., Zhang X.P. J. Org. Chem. 2003, 68, 6215.

3. Chen Y., Zhang X.P. J. Org. Chem. 2003, 68, 4432.

4. Takanami T., Hayashi M., Hino F., Suda K. Tetrahedron Lett. 2003, 44, 7353.

5. Esdaile L.J., Senge M.O., Arnold D.P. Chem. Commun. 2006, 4192.

6. Gao G.Y., Colvin A.J., Chen Y., Zhang X.P. Org. Lett. 2003, 5, 3261.

7. Gao G.Y., Colvin A.J., Chen Y., Zhang X.P. J. Org. Chem. 2004, 69, 8886

8. Atefi F., McMurtrie J.C., Turner P., Duriska M., Arnold D.P. Inorg. Chem. 2006, 45, 6479.

9. Shi B., Boyle R.W. J. Chem. Soc., Perkin Trans. 1 2002, 1397.

10. Chang J.C., Ma C.J., Lee G.H., Peng S.M., Yeh C.Y. Dalton Trans. 2005, 1504.

11. Pereira M.M., Muller G., Ordinas J.I., Azenha M.E., Arnaut L.G. J. Chem. Soc., Perkin Trans. 2 2002, 1583.

12. Hata H., Shinokubo H., Osuka A. J. Am. Chem. Soc. 2005, 127, 8264.

13. Cunha A.C., Gomes A.T.P.C., Ferreira V.F., de Souza M.C.B.V., Neves M.G.P.M.S., Tome A.C., Silva A.M.S., Cavaleiro J.A.S. Synthesis 2010, 510 .

14. Hyslop A.G., Kellett M.A., Iovine P.M., Therein M.J. J. Am. Chem. Soc. 1998, 120, 12676.

15. Tremblay-Morin J.P., Ali H., van Lier J.E. Tetrahedron Lett. 2006, 47, 3043.

16. Ali H., van Lier J.E. Tetrahedron Lett. 2009, 50, 1113.

17. Cheng F.Y., Zhang S., Adronov A., Echegoyen L., Diederich F. Chem. Eur. J. 2006, 12, 6062.
18. Peng X.B., Nakamura Y., Aratani N., Kim D., Osuka A. Tetrahedron Lett. 2004, 45, 4981.

19. Yu L., Lindsey J.S. Tetrahedron 2001, 57, 9285.

20. Wagner R.W., Johnson T.E., Li F., Lindsey J.S. J. Org. Chem. 1995, 60, 5266.

21. Severac M., Le Pleux L., Scarpaci A., Blart E., Odobel F. Tetrahedron Lett. 2007, 48, 6518.

22. Shen D.M., Liu C., Chen Q.Y. Eur. J. Org. Chem. 2007, 1419.

23. Punidha S., Sinha J., Kumar A., Ravikanth M. J. Org. Chem. 2008, 73, 323

24. Shetti V.S., Ravikanth M. Eur. J. Org. Chem. 2010, 494.

25. Huang C.Y., Su Y.O. Dalton Trans. 2009, 39, 8306.

26. Sugiura K., Fujimoto Y., Sakata Y. Chem. Commun. 2000, 1105 .

27. Hisaki I., Hiroto S., Kim K.S., Noh S.B., Kim D., Shinokubo H., Osuak A. Angew Chem. Int. Ed. 2007, 46, 5125.

28. Mikhalitsyna E.A., Tyurin V.S., Zamylatskov I.A., Khrustalev V.N., Beletskaya I.P. Dalton Trans. 2012, 41, 7624.

29. Mikhalitsyna E.A., Tyurin V.S., Khrustalev V.N., Lonin I.S., Beletskaya I.P. Dalton Trans. 2014, 43, 3563.

30. Polevaya Yu.P., Tyurin V.S., Beletskaya I.P. J. Porphyrins Phthalocyanines 2014, 18, 20.

31. Ukai T., Kawazura H., Ishii Y., Bonnet J.J., Ibers J.A. $J$. Organomet. Chem. 1974, 65, 253.

32. Mikhalitsyna E.A., Tyurin V.S., Nefedov S.E., Syrbu S.A., Semeikin A.S., Koifman O.I., Beletskaya I.P. Eur. J. Inorg. Chem. 2012, 36, 5979.

33. Yakushev A.A., Averin A.D., Anokhin M.V., Maloshitskaya O.A., Lamaty F., Beletskaya I.P. Macroheterocycles 2014, 7, 358-364.

34. Anokhin M.V., Averin A.D., Beletskaya I.P. Eur. J. Org. Chem. 2011, 6240.

35. Panchenko S.P., Averin A.D., Anokhin M.V., Maloshitskaya O.A., Beletskaya I.P. Beilstein J. Org. Chem. 2015, 11, 2297.

36. Uglov A.N., Averin A.D., Buryak A.K., Beletskaya I.P. ARKIVOC 2011, viii, 99.

37. Uglov A.N., Averin A.D., Buryak A.K., BessmertnykhLemeune A., Guilard R., Beletskaya I.P. Macroheterocycles 2013, 6,33 .

38. Chandrasekhar V., Das S., Yadav R., Hossain S., Parihar R., Subramaniam G., Sen P. Inorg. Chem. 2012, 51, 8664.

39. Espada-Bellido E., Dolores Galindo-Riano M., Garcia-Vargas M., Narayanaswamy R. Applied Spectrosc. 2010, 64, 727.

40. Liu S.-R., Wu Sh.-P. J. Fluoresc. 2011, 21, 1599.

41. Huang L., Cheng J., Xie K., Xi P., Hou F., Li Z., Xie G., Shi Y., Liu H., Bai D., Zeng Z. Dalton Trans. 2011, 40, 10815.

42. Liu Z., Zhang Ch., Wang X., He W., Guo Z. Org. Lett. 2012, $14,4378$.

43. Kumar M., Kumar N., Bhalla V. Dalton Trans. 2012, 41, 10189.

44. Wu Sh.-P., Du K.-J., Sung Y.-M. Dalton Trans. 2010, 39, 4363.

45. Ranyuk E., Uglov A., Meyer M., Bessmertnykh-Lemeune A., Denat F., Averin A., Beletskaya I., Guilard R. Dalton Trans. 2011, 40, 10491

46. Kaur P., Kaur M., Singh K. Talanta 2011, 85, 1050.

47. Liu M.-X., Wei T.-B., Lin Q., Zhang Y.-M. Spectrochim. Acta, Part A 2011, 79, 1837.

48. Sheng R., Wang P., Gao Y., Wu Y., Liu W., Ma J., Li H., Wu Sh. Org. Lett. 2008, 10, 5015.

49. Tan J., Yan X.-P. Talanta 2008, 76, 9.

50. Kaur P., Kaur S., Singh K., Sharma P.-R., Kaur T. Dalton Trans. 2011, 40, 10818.

51. Sikdar A., Panja S.S., Biswas P., Roy S. J. Fluoresc. 2012, 22 , 443. 